\title{
Telomerase activity in cervical smears
}

\author{
G. Saretzki ${ }^{\text {a }}$, H. Fischer ${ }^{\text {b }}$, I.-G. Kaufmann ${ }^{\text {a }}$, \\ C. Schewe ${ }^{\text {a }}$, B. Nadjari ${ }^{\text {a }}$, J. Blohmer ${ }^{\mathrm{b}}$ and \\ St. Hauptmann ${ }^{\text {a,* }}$ \\ a Institute of Pathology, Charité Hospital Berlin, \\ Schumannstrasse 20/21, D-10117 Berlin, Germany \\ ${ }^{\mathrm{b}}$ Department of Gynecology, Charité Hospital Berlin, \\ Schumannstrasse 20/21, D-10117 Berlin, Germany
}

Received 15 March 2001

Revised 13 August 2001

It is well known that almost all carcinoma cells including those of the uterine cervix have re-established their telomerase activity. However, until now there is no conclusive picture on the telomerase activity in cervical dysplasias and about their relationship to HPV infection. To investigate this question, material from 34 patients (15 with normal epithelium, 11 with LGSIL, 8 with HGSIL) obtained by conventional cervical brushing was used and subjected to non-radioactive TRAP-ELISA (Boehringer Mannheim). The HPV analysis was performed by PCR on formalin-fixed, paraffin-embedded biopsy material obtained after cytological investigation. We could show that telomerase activity is detectable in normal cervical epithelium, and that an gradual increase exists for both telomerase activity and HPV positivity from normal epithelium to HGSIL. However, HPV infection and telomerase activity appear to be independent of each other. The high frequency of telomerase positivity in patients with normal cervical epithelium indicates that telomerase activity is not a useful differential diagnostic aid. Whether patients with telomerase-positive dysplasias have a higher probability to progress into an invasive carcinoma remains to be clarified by follow-up studies.

Keywords: Cervix uteri, dysplasia, telomerase activity, HPV infection, gynecological cytology

\section{Introduction}

In normal somatic cells, other than those belonging to the stem cell compartment, the telomeric ends of chromosomes are shortened after each round of replication. This shortening happens because the telom-

\footnotetext{
${ }^{*}$ Corresponding author.
}

erase, an enzyme capable of elongating the telomers, is silenced in these cells. After a critical telomer length has been achieved, cells stop cycling, a phenomenon called replicative senescence. In contrast to normal cells, telomerase activity has been detected in the majority of malignant neoplasia, suggesting that reactivation or overexpression of telomerase is involved in carcinogenesis $[3,11,13,21,22]$. In invasive squamous cell carcinomas of the cervix uteri, telomerase activity is shown in nearly all cases $[1,14,19,23,26]$. Because this enzyme was found to be reactivated even in some pre-invasive lesions $[13,20]$ it was thought that it could serve as an useful diagnostic tool to detect early malignant stages, especially if minimal-invasive cytological sampling techniques were used, such as fine needle biopsies, peritoneal, lung and bladder washings and gynecological smears $[5,7,10,14,24,25,29]$.

The aim of the present study was to analyze the frequency of telomerase positivity in low-grade and high-grade squamous intraepithelial lesions (LGSIL and HGSIL, resp.) of the cervical epithelium compared to cervical epithelium, and to ask whether there is a correlation between HPV infection and telomerase activity.

Our results indicated that telomerase activity was found in more than a quarter of normal epithelium of the cervix uteri, diminishing its use as differential diagnostic aid. On the other hand, although HPV infection and telomerase positivity were found to be independent from each other there was a clear gradual increase of both from normal epithelia to HGSIL.

\section{Material and methods}

\subsection{Patients and sample collection}

Thirty four patients with normal or dysplastic changes, randomly selected from the oncogynecological dispensary at the Charite hospital, were investigated. Smears were collected from the ectocervix with a wooden spatula and from endocervical by brushing in each case. Two parallel probes from both localizations were obtained: the first - for telomerase investigation 
- was transferred to PBS and kept for a maximum of 4-6 hours (mean 2.5 hours) at $4{ }^{\circ} \mathrm{C}$. Afterwards cells were centrifuged and the pellet was stored at $-80^{\circ} \mathrm{C}$ until analysis. The second sample was prepared for cytological diagnosis.

\subsection{Cytological and histological investigations}

Cervical smears were fixed in ethanol, stained with Papanicolaou and examined by a gynecologist. Histological examination and analysis of HPV infection were performed on cervical punch or cone biopsies, which were fixed in $10 \%$ neutral-buffered formalin, embedded in paraffin, cut at a thickness of $5 \mu \mathrm{m}$, and stained with hematoxylin \& eosin. Histological diagnosis was done by pathologists. Indications to obtain a biopsy were either repeated cytological diagnoses of dysplasia, repeated cytological abnormalities in patients with HPV positivity, or cone biopsy due to dysplasia in their history and abnormal colposcopy. The patient informed consent had been obtained in all cases.

\subsection{TRAP (telomeric repeat amplification protocol) assay}

Detection of telomerase activity was performed using the non-radioactive Telomerase-PCR-ELISA (Boehringer, Mannheim). The pellets were resuspended in Chaps-buffer and the cell number was determined by counting in a hemocytometer. If possible, the cell concentration was adjusted to an optimum of $10^{6}$ cells per $200 \mu$ l Chaps buffer [11]. The minimal cell number used was $5 \times 10^{3}$. In cases with a low cell number the template volume used for the PCR was enhanced up to $10 \mu \mathrm{l}$. The PCR and the following detection of the synthesized telomeric repeats with a non-radioactively labeled probe were performed according to the manufacturer's instructions (Boehringer Mannheim), except that $10 \mu \mathrm{l}$ (instead of $5 \mu \mathrm{l}$ ) of the PCR product were used for denaturation to enhance the sensitivity of the assay. Each sample was analyzed in duplicate. Chaps-buffer without cells and cell extracts from healthy persons were used as negative controls. Analysis was repeated at least once in an independent test. Samples were regarded as telomerase-positive, if the detected calorimetric value in the reaction with the enzyme linked antibody against the labeled probe was at least twice the value of the negative control. The sensitivity of the assay was determined using HL-60 cells which are known to have high telomerase activity. Specificity of the telomerase reaction was determined by heat inactivation of samples in parallel PCR reactions.

\subsection{HPV-detection}

DNA was prepared from formalin-fixed, paraffinembedded tissue. The quality of the DNA preparation was tested by amplification of a genomic sequence of -globin. For detection of a wide array of HPV variants (e.g., HPV-types 6, 11, 16, 18, 31, 33, 35, 42, $43,44,45,51,52,56)$ we used the consensus primer pair MY 09 (primer for the negative strand) and MY11 (primer for the positive strand) for amplification of the L1 region (Digene, Beltsville, USA). The specificity of the products and their typing were established with a sensitive non-radioactive method using the Sharp Signal System (Digene, Beltsville, USA) via hybridization with a specific single-stranded RNA probe to the $5^{\prime}$ biotinylated PCR product and reaction with an enzyme linked anti-hybrid antibody assay at $405 \mathrm{~nm}$.

\section{Results}

\subsection{Detection threshold}

To determine the detection threshold and the sensitivity of the non-radioactive TRAP-ELISA, we used HL-60 cells which are known to have high telomerase activity. The assay was positive if the sample contained at least 50 tumor cells (a $1: 200$ dilution of a standard lysate concentration of $10^{4}$ tumor cells in $2 \mu \mathrm{l}$ ). Telomerase activity is still detectable in a sample with a total cell number of $10^{4}$ squamous epithelial cells. Thus, the method has a sensitivity of around $0.5 \%$ cells with high telomerase activity, and there was obviously no inhibition of the TRAP assay due to telomerase negative cells as reported by other groups as well [14]. To exclude an influence of storage, we harvested the tumor cells for $2,6,18$, and $24 \mathrm{~h}$ in $\mathrm{PBS}$ at $4{ }^{\circ} \mathrm{C}$. No decrease in telomerase activity could be detected.

\subsection{Normal samples}

We had samples from 15 patients with normal epithelium. In 3 samples (20\%) there was a HPV (high risk types) infection, and in 5 samples (33.3\%) telomerase activity was detectable (Fig. 1). Only one of the three HPV positive cases had telomerase activity, and 4 of the cases with telomerase positivity were HPV negative. In only one case HPV and telomerase positivity were associated (Fig. 2). Therefore, no association was found between HPV positivity and telomerase activity in normal cervical epithelium. In 4 patients there was 


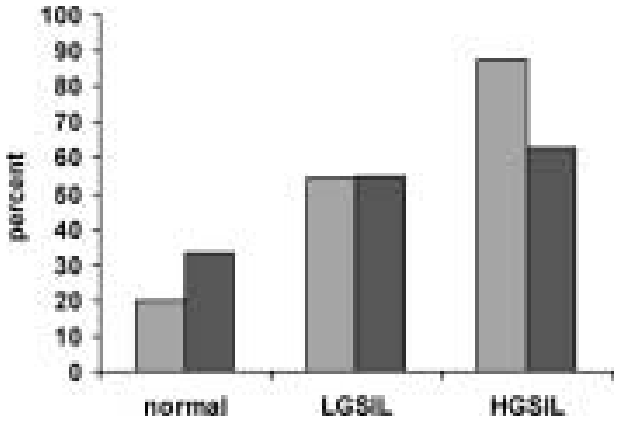

Fig. 1. HPV-positivity (blue) and telomerase activity (red) in normal cervical epithelium and squamous intraepithelial lesions, low grade (LGSIL), and high grade (HGSIL).

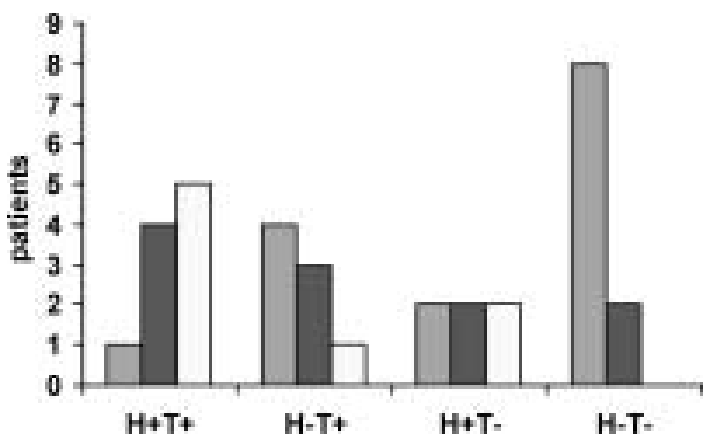

Fig. 2. Association between HPV positivity $(\mathrm{H}+)$ and telomerase activity $(\mathrm{T}+)$ in normal cervical epithelium (blue) and squamous intraepithelial lesions, low grade (LGSIL; red), and high grade (HGSIL; yellow).

a history of LGSIL and HGSIL, respectively. Three of them had telomerase activity, and in 2 patients HPV was still detectable despite the lack of morphological abnormalities.

\subsection{Dysplastic lesions}

Nineteen patients with dysplasias (11 cases with LGSIL, 8 cases with HGSIL) were analyzed. In 6 cases $(54.5 \%)$ with LGSIL, and in 5 cases $(62.5 \%)$ with HGSIL telomerase activity was detected. HPV positivity was found in 6 patients $(54.5 \%)$ with LGSIL and in 7 patients $(87.5 \%)$ with HGSIL (Fig. 1). Whereas the increase in HPV positivity from normal epithelium to LGSIL and HGSIL was statistical significant $(p=0.007)$, that of telomerase positivity was not. Taking all patients with dysplastic lesions together there were 8 patients (42\%) with HPV positivity and telomerase activity, whereas the reverse case was found in only 3 patients (16\%). In 3 patients telomerase activity was found without HPV positivity, while the other combination occurred in 5 patients (26\%) (Fig. 2). Again, no significant association exists between HPV infection and telomerase positivity. There was no relationship between the degree of telomerase activity and the degree of dysplasia and HPV infection, respectively.

\section{Discussion}

Activation of telomerase, an enzyme that synthesizes the telomere ends of chromosomes, has been implicated in immortalization of somatic cells, and, therefore, in the pathogenesis of human malignancies [3, $11,21,20]$. For invasive carcinomas of the cervix uteri telomerase, activity has been reported in $88-100 \%$ of cases $[1,14,23,26]$. The $58 \%$ of telomerase positivity in SIL found in this study lies between the very broad range of $25-96 \%$ described in the literature $[10,14,19$, $23,24,27]$. Despite the frequency of telomerase activity in SIL, the telomerase reverse transcriptase has been detected in a recent study only once $(6 \%)$, whereas more than $80 \%$ of cases possessed telomerase RNA component [16]. Nevertheless, the lack of detectable telomerase activity in more than one third of SIL in this study is remarkable high. Technical reasons are unlikely because HL-60 cells, which are known to posses high telomerase activity, did not show any loss of activity despite storage at $4{ }^{\circ} \mathrm{C}$ for up to 24 hours. Moreover, Duggan et al. [7] were able to detect telomerase activity within samples stored for 7 days at room temperature, indicating that telomerase is not as sensible as originally assumed. On the other hand, one could speculate that the cotton swabs used to collect the samples contain telomerase-inhibiting substances. This, however, is not likely because other investigators [10,14] collect their samples in exact the same way without any detectable negative influence. We cannot exclude that the number of dysplastic cells within the negative samples are below $0.5 \%$, although the Pap smears which were obtained after sampling for telomerase analysis contained plenty of dysplastic cells. Therefore, we suggest that dysplasias exist with very low or even without telomerase activity. The important question is whether dysplasias without telomerase activity represent lesions with a low or even negligible risk of progression into a high grade lesion. From the cytological literature, it is known that only $16 \%$ of LGSIL will show progression, whereas $37 \%$ persist and $47 \%$ regress spontaneously [28]. Therefore, follow-up studies are necessary to clarify whether telomerase activity 
are indeed early markers of an enhanced risk of malignant progression. In our study the degree of dysplasia was not correlated to telomerase activity as reported by other authors [14,19,31], although there are also other reports in the literature [10,16,23,24].

Dysplasia of the cervical squamous epithelium is frequently associated with HPV infection of the high risk type such as HPV16 and HPV18. The oncogenic activities of HPV are complex but may involve telomerase activation. This has been shown by Klingelhutz et al. [12] who found an activation of telomerase in HPV-transfected keratinocytes with genomic integration of the viral DNA. However, in dysplastic lesions HPV is present mostly in the episomal state [6], but the relationship between non-integrated HPV and telomerase activity has not been studied yet. Telomerase activity, in the absence of morphological detectable signs of HPV infection in patients with a history of HPVassociated lesion as found in this investigation, has also been described also by Iwasaka et al. [10]. Therefore, telomerase activity could be a sensitive marker of a persisting but hidden HPV infection which has to be clarified in a prospective follow-up study. On the other hand, it cannot be excluded that simply the proliferation induced by viral infection may be the reason of telomerase activity, because even lesions which are associated with infection by HPV of low risk type (e.g., Condyloma accuminatum) are telomerase positive as shown in this and other studies [17,23].

This investigation demonstrates that telomerase activity is not a specific marker for premalignant changes of the cervical epithelium, since it is detectable in up to one third of normal epithelium. Because the material investigated in this study were from patients with repeated cytological abnormalities one can expect a somewhat lower frequency in normal smears from a general population. On the other hand, the presence of activated lymphocytes or granulocytes can also induce a positive result because both have active telomerase $[8,18]$. Moreover, even physiological proliferative processes (e.g., proliferating endometrium) are associated with telomerase activity $[2,4,15,30]$, and stem cells in renewable tissues like skin and intestine were also shown to be telomerase positive $[9,13]$. Therefore, all types of regenerative epithelial changes may be associated with telomerase activity.

Taken together our data indicate that telomerase activity is not helpful in differential diagnosis of dysplastic lesions. Whether dysplasias without detectable telomerase activity have a lower risk for progression than those with telomerase activity, and whether telom- erase activity in the absence of any morphological changes indicates a persistent HPV infection, remains to be clarified in follow-up studies.

\section{References}

[1] S.K. Anderson, J. Shera, L. Ihle et al., Telomerase activation in cervical cancer, Am. J. Pathol. 151 (1997), 25-31.

[2] C. Belair, T.R. Yeager, P.M. Lopez et al., Telomerase activity: a biomarker of cell proliferation, not malignant transformation, Proc. Natl. Acad. Sci. USA 94 (1997), 13,677-13,682.

[3] A.G. Bodnar, M. Oulette, M. Frolkis et al., Extension of life span by introduction of telomerase into normal human cells, Science 279 (1998), 349-352.

[4] T.P. Brien, B.V. Kallakury, C.V. Lowry, R.A. Ambros, P.J. Muraca, J.H. Malfetano and J.S. Ross, Telomerase activity in benign endometrium and endometrial carcinoma, Cancer Res. 57 (1997), 2760-2764

[5] G. Dalbagni, W. Han, Z.-F. Zhang et al., Evaluation of telomeric repeat amplification protocol (TRAP) assay for telomerase as a diagnostic modality in recurrent bladder cancer, Clin. Cancer Res. 3 (1997), 1593-1598.

[6] B. Daniel, G. Mukhererjee, L. Seshadri et al., Changes in the physical state and expression of human papillomavirus type 16 in the progression of cervical intraepithelial neoplasia lesions analysed by PCR, J. Gen. Virol. 76 (1995), 2589-2593.

[7] B.D. Duggan, M. Wan, M. Yu et al., Detection of ovarian cancer cells: comparison of a telomerase assay and cytologic examination, J. Natl. Cancer Inst. 90 (1998), 238-242.

[8] K. Hiyama, Y. Hirai, S. Kyozumi et al., Activation of human lymphocytes and hematopoietic progenitor cells, J. Immunol. 155 (1995), 3712-3715.

[9] C. Hõrle-Bachor and P. Boukamp, Telomerase activity in the regenerative basal layer of the epidermis in human skin and in immortal and carcinoma-derived skin keratinocytes, Proc. Natl. Acad. Sci. USA 93 (1996), 6476-6481.

[10] T. Iwasaka, P.S. Zheng, M. Yokoyama et al., Telomerase activation in cervical neoplasia, Obstet. Gynecol. 91 (1998), 260262.

[11] N.W. Kim, M. Piatyszek, Prowse et al., Specific association of human telomerase activity with immortal cells and cancer, Science 266 (1994), 2011-2015.

[12] A.J. Klingelhutz, S.A. Foster and J.K. McDougall, Telomerase activation by the E6 gene product of human papillomavirus type 16, Nature 380 (1996), 79-81.

[13] K.A. Kolquist, L.W. Ellisen, C.M. Counter et al., Expression of TERT in premalignant lesions and a subset of cells in normal tissue, Nat. Genet. 19 (1998), 182-186.

[14] S. Kyo, M. Takakura, H. Ishikawa et al., Application of telomerase assay for the screening of cervical lesions, Cancer Res. 57 (1997), 1863-1867.

[15] S. Kyo, M. Takakura, T. Kohama and M. Inoue, Telomerase activity in human endometrium, Cancer Res. 57 (1997), 610614.

[16] S. Lanham, A. Herbert and P. Watt, HPV detection and measurement of HPV-16, telomerase, and survivin transcripts in colposcopy clinic patients, J. Clin. Pathol. 54 (2001), 304-308. 
[17] A. Mutirangura, V. Sriuranpong, W. Termrunggraunglert et al., Telomerase activity and papillomavirus in malignant, premalignant and benign cervical lesions, Br. J. Cancer 78 (1998), 933-939.

[18] K.F. Norrback and G. Roos, Telomeres and telomerase in normal and malignant haemopoetic cells, Eur. J. Cancer 33 (1997), 774-780.

[19] Ch. Pao, C.-J. Tseng, C.-Y. Lin et al., Differential expression of telomerase activity in human cervival cancer and cervical intraepithelial neoplastic lesions, J. Clinic. Oncol. 15 (1997), 1932-1937.

[20] J.W. Shay and S. Bacchetti, A survey of telomerase activity in human cancer, Eur. J. Cancer 33 (1997), 787-792.

[21] J.W. Shay, Telomerase in human development and cancer, J. Cell Physiol. 173 (1997), 266-270.

[22] J.W. Shay and W.E. Wright, The reactivation of telomerase activity in cancer progression, Trends Genet. 12 (1996), 129-130.

[23] K. Shroyer, L.C. Thompson, T. Enomoto et al., Telomerase expression in normal epithelium, reactive atypia, squamous dysplasia, and squamous cell carcinoma of the uterine cervix, Am. J. Clin. Pathol. 109 (1998), 153-162.

[24] P.J. Snijders, M. van Diun, J.M. Walboomers et al., Telomerase activity exclusively in cervical carcinomas and a subset of cervical intraepithelial neoplasia grade III lesions, Cancer Res. $\mathbf{5 8}$ (1998), 3812-3818.
[25] T. Sugino, K. Yoshida, J. Bolodeoku et al., Telomerase activity in human breast cancer and benign breast lesions: Diagnostic applications in clinical specimens including fine needle aspirates, Int. J. Cancer 69 (1996), 301-306.

[26] M. Takakura, S. Kyo, T. Kanaya et al., Expression of human telomerase subunit and correlation with telomerase activity in cervical cancer, Cancer Res. 58 (1998), 1558-1561.

[27] G.B. Wisman, H. Hollema, S. de Jong et al., Telomerase activity as a biomarker for (pre) neoplastic cervical disease in scrapings and frozen sections from patients with abnormal cervical smear, J. Clin. Oncol. 16 (1998), 2238-2245.

[28] T.C. Wright, R.J. Kurman and A. Ferenczy, Precancerous Lesions of the Cervix, 4th edn, Springer-Verlag, New York, 1994, pp. 229-277. (R.J. Kurman ed. Blaustein's pathology of the female genital tract.)

[29] K. Yashima, R. Ashfaq, J. Nowak et al., Telomerase activity and expression of its RNA component in cervical lesions, Cancer 82 (1998), 1319-1327.

[30] S. Yasumoto, C. Kunimura, K. Kikuchi et al., Telomerase activity in normal epithelial cells, Oncogene 13 (1996), 433-439.

[31] P.S. Zheng, T. Iwasaka, M. Yokoyama et al., Telomerase activation in in vitro and in vivo cervical carcinogenesis, Gynecol. Oncol. 66 (1997), 222-226. 


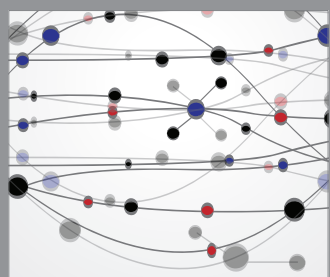

The Scientific World Journal
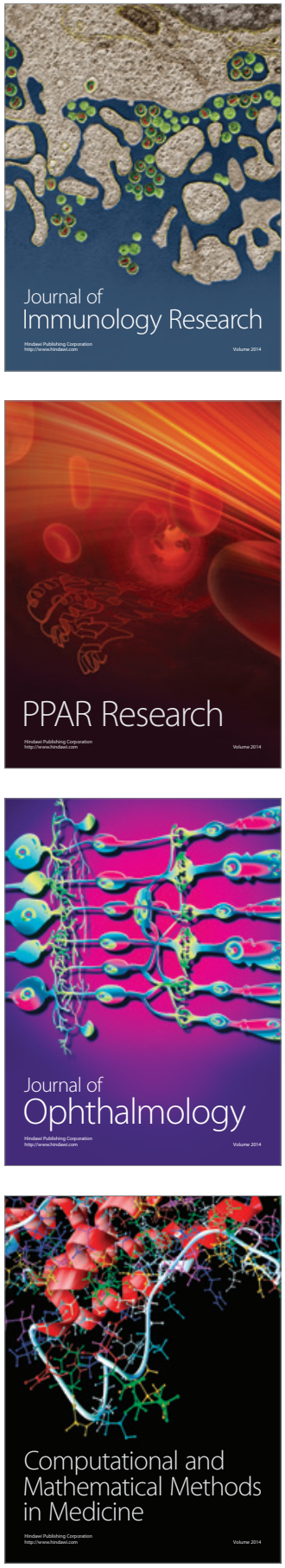

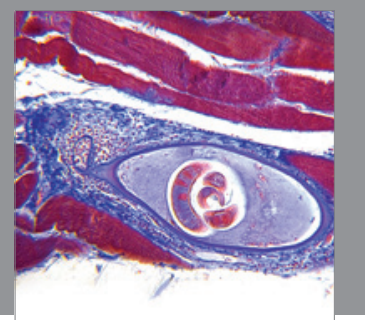

Gastroenterology

Research and Practice
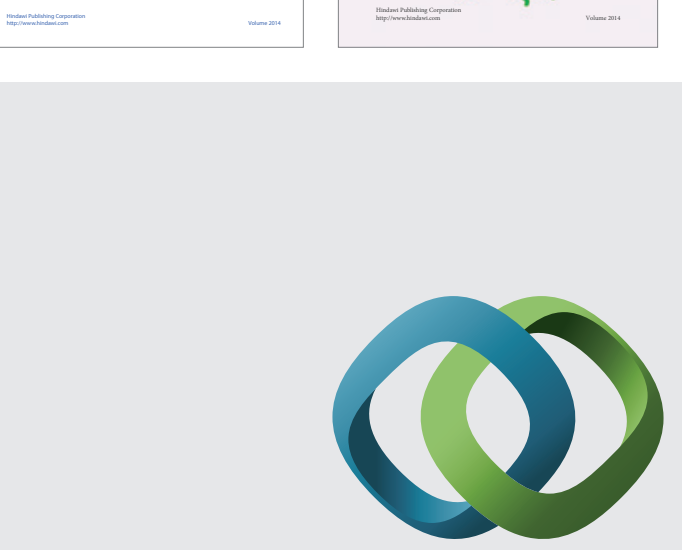

\section{Hindawi}

Submit your manuscripts at

http://www.hindawi.com
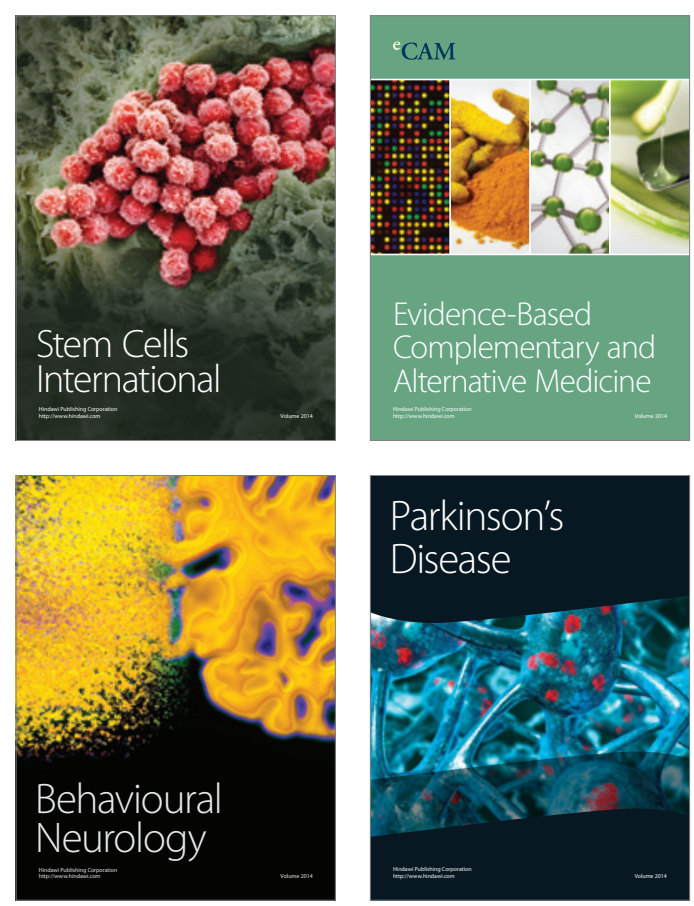

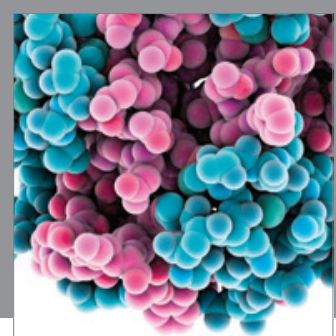

Journal of
Diabetes Research

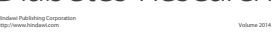

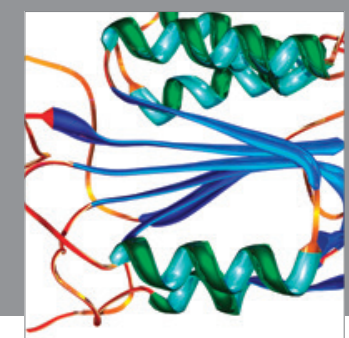

Disease Markers
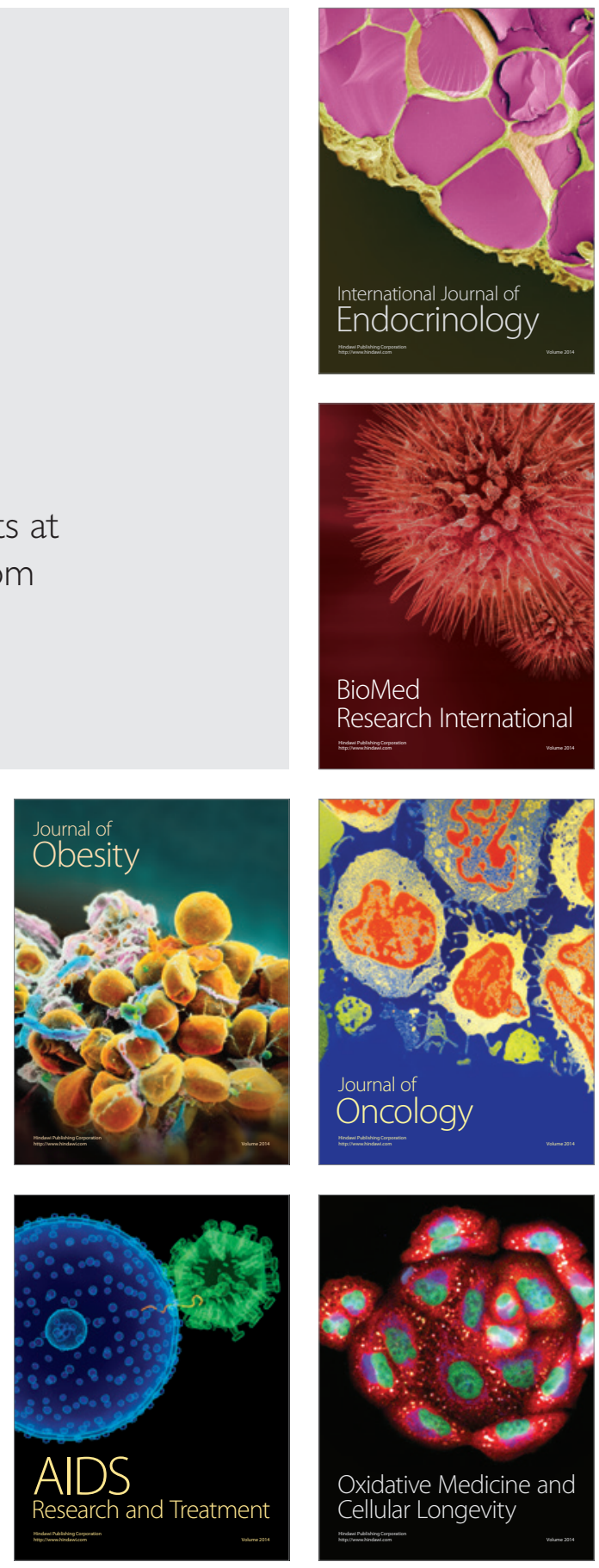